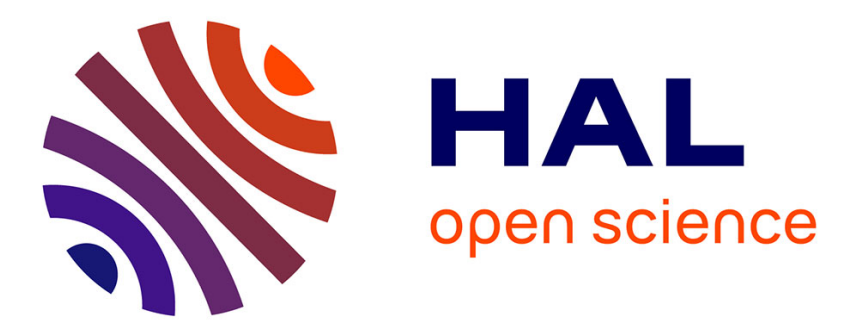

\title{
Polymorphism of cyclopentanol: Crystallographic characterization of the ordered and disordered phases
}

M. A. Rute, Josep Salud, David O. Lopez, Josep Lluis Tamarit, Philippe

Négrier, Maria Barrio, Denise Mondieig

\section{- To cite this version:}

M. A. Rute, Josep Salud, David O. Lopez, Josep Lluis Tamarit, Philippe Négrier, et al.. Polymorphism of cyclopentanol: Crystallographic characterization of the ordered and disordered phases. Chemistry of Materials, 2003, 15 (25), pp.4725-4731. 10.1021/cm031081b . hal-01550888

\section{HAL Id: hal-01550888 \\ https://hal.science/hal-01550888}

Submitted on 3 Jan 2018

HAL is a multi-disciplinary open access archive for the deposit and dissemination of scientific research documents, whether they are published or not. The documents may come from teaching and research institutions in France or abroad, or from public or private research centers.
L'archive ouverte pluridisciplinaire HAL, est destinée au dépôt et à la diffusion de documents scientifiques de niveau recherche, publiés ou non, émanant des établissements d'enseignement et de recherche français ou étrangers, des laboratoires publics ou privés.

\section{다(1)(2)}

Distributed under a Creative Commons Attribution - ShareAlikel 4.0 International 


\title{
Polymorphism of Cyclopentanol: Crystallographic Characterization of the Ordered and Disordered Phases
}

\author{
M. A. Rute, ${ }^{\dagger}$ J . Salud, ${ }^{\dagger}$ D. O. López, ${ }^{\dagger}$ J . LI. Tamarit, ${ }^{*},{ }^{\dagger}$ Ph. Negrier, ${ }^{\ddagger}$ \\ M. Barrio, ${ }^{\dagger}$ and D. Mondieig ${ }^{\ddagger}$ \\ Departament de Física i Enginyeria Nuclear, E.T.S.E.I.B., Universitat Politècnica de \\ Catalunya, Catalonia, Spain, and Centre de Physique Moléculaire, Optique et Hertzienne, \\ UMR 5798 au CNRS-Université Bordeaux I, France
}

\begin{abstract}
The polymorphism of cyclopentanol $\left(\mathrm{C}_{5} \mathrm{H}_{10} \mathrm{O}\right)$ has been further investigated by $\mathrm{X}$-ray powder diffraction experiments and it has been found to agree with the most recent thermodynamic study [J. Chem. Thermodyn. 1995, 27, 953]. In addition to the previously reported orientationally disordered hexagonal phases I and II, the lattice symmetry of the lowtemperature or dered phases III and IV have been determined by means of pattern-matching analyses both as monoclinic with $Z=24$ (Cc and C2/c, respectively). The patterns revealed no significant differences between phases III and IV and the transition between both phases was found through careful determination of the variation of the lattice parameters as a function of the temperature. The strength of intermolecular interactions as well as the anisotropy in the solid phases has been analyzed by the study of the isobaric thermalexpansion tensor and the results are discussed in the light of previously reported literature covering dynamics disorder.
\end{abstract}

\section{Introduction}

For years much attention has been paid to reorientational motion in the solid state. Such a particular orientational disorder was first described by Timmermanns in $1961^{1}$ who showed that crystals composed of molecules more or less spherical in shape have small entropy and volume changes of fusion. These compounds, named plastic crystals or "orientationally disordered crystals" (ODIC), ${ }^{2}$ display at least one additional transition accompanying a relatively large entropy change, in such a way that crystals melt in successive steps, the rotational degrees melting at the ordereddisordered low-temperature phase transition.

The lower order cyclic alcohols are examples of pseudoglobular molecules displaying ODIC phases. ${ }^{3-5}$ In addition, these molecules can display a finite number of molecular conformations, giving rise to a rich polymorphism. The ring of cyclic molecules displays several puckered conformations, the number of which increases with the number of carbons involved in the ring. ${ }^{6,7}$

* To whom correspondence should be addressed. Dr. J . LI. Tamarit, Departament de Física i Enginyeria Nuclear ETSEIB, Universitat Politècnica de Catalunya Diagonal, 64708028 Barcelona, Catalonia, Spain. Phone +34 934016564 . Fax +34 9340118 39. E-mail: J OSE.LUIS.TAMARIT@UPC.ES.

† Universitat Politècnica de Catalunya.

₹ CNRS-Université Bordeaux I.

(1) Timmermans, J . J . Chim. Phys. 1938, 35, 331

(2) Stavely, L. A. K. Annu. Rev. Phys. Chem. 1962, 13, 351.

(3) Fuchs, A. H.; Virlet, J. R.; Andre, D.; Szwarc, H. J . Chim. Phys.

$1985,82,293$.

(4) Anderson, O.; Ross, R. G. Mol. Phys. 1990, 71 (3), 523.

(5) Shablakh, M.; Dissado, L. A.; Hill, R. M. J . Chem. Soc., Faraday Trans. 2 1983, 79, 369.

(6) Lambert, J . B.; Xue, L.; Howton, S. C. J . Am. Chem. Soc. 1991, $113,9858$.

(7) Ekejiuba, I. O. C.; Hallan, H. E. J . Chem. Soc. B. 1970, 209.
Figure 1 displays the two puckered conformations of cyclopentanol, the envelope and the half-chair. In addition to the ring conformation, the polar $\mathrm{OH}$ group may adopt either of two conformations, the axial or the equatorial, with respect to the carbon atom on which the group is bonded. ${ }^{8}$ It should be pointed out that such a hydroxyl group orientational disorder can exist without affecting necessarily the global packing of the solid state structure and thus enabling the existence of an orientationally disordered (OD) phase.6,7,9 Moreover, although organic crystal structures are mainly controlled by steric effects, the existence of the $\mathrm{OH}$ group gives rise to a hydrogen-bonding interaction, which certainly contributes to the stabilization of a preferred crystal structure. ${ }^{10}$ This effect is especially interesting when OD phases are considered because compatibility among disorder, molecular interactions, and lattice symmetry is needed. 11,12

As far as cyclopentanol is concerned (hereinafter referred to as $\mathrm{c}^{-} \mathrm{C}_{5} \mathrm{H}_{10} \mathrm{O}$ ), its polymorphism has been studied earlier by several methods such as calorimetry at normal ${ }^{13-15}$ and high pressure, ${ }^{16}$ infrared $^{7-9}$ and diel ectric spectroscopy5,17-21 techniques, high-pressure

(8) Allinger, M. C.; Hirsh, J . A.; Miller, M. A.; Tyminski, I. J . J Am. Chem. Soc. 1961, 90, 1197.

(9) Ściesińska, E.; Ściesiński, J .; Godlewska, M. Spectrosc. Lett. 1982, 15 (5), 399.

(10) Salud, J.; Barrio, M.; López, D. O.; Tamarit, J . LI.; Alcobé, X. J . Appl. Crystallogr. 1998, 31, 748.

(11) Barrio, M.; López, D. O.; Tamarit, J . LI.; Negrier P.; Haget, Y. J . Mater. Chem. 1995, 5 (3), 431.

(12) Barrio, M.; López, D. O.; Tamarit, J . LI.; Negrier P.; Haget, Y. J . Solid State Chem. 1996, 124, 29.

(13) Adachi, K.; Suga, H.; Seki, S. Bull. Chem. Soc. J pn. 1970, 43, 1916.

(14) Parks, G. S.; Kennedy, W. D.; Gates, R. R.; Mosely, J . R.; Moore C. E.; Renquist, M. L. J . Am. Chem. Soc. 1956, 78, 56. 
Table 1. Polynomial Equations $\mathbf{P}=\mathbf{p}_{0}+\mathbf{p}_{1} \mathbf{T}+\mathbf{p}_{2} \mathbf{T}^{2}(\mathbf{T}$ in $\mathrm{K})$ Corresponding to the Lattice Parameters $\mathbf{p}$ (in $\AA$ ) of the C- $\mathrm{C}_{5} \mathrm{H}_{10} \mathrm{O}^{\mathrm{a}}$

\begin{tabular}{|c|c|c|c|c|c|c|}
\hline phase & temp range & parameter & $p_{o}$ & $p_{1} \times 10^{3}$ & $\mathrm{p}_{2} \times 10^{5}$ & $\mathrm{R} \times 10^{5}$ \\
\hline IV & $93-176$ & $\begin{array}{l}\mathrm{a} \\
\mathrm{b} \\
\mathrm{c} \\
\beta\end{array}$ & $\begin{array}{c}23.30(3) \\
6.271(6) \\
21.67(46) \\
99.61(7)\end{array}$ & $\begin{array}{c}0.7(4) \\
-0.36(9) \\
-7.3(7) \\
13.2(1.0)\end{array}$ & $\begin{array}{l}1.36(15) \\
0.38(3) \\
4.2(2) \\
-6.1(3)\end{array}$ & $\begin{array}{r}8 \\
6 \\
13 \\
4\end{array}$ \\
\hline III & $176-202$ & $\begin{array}{l}\mathrm{a} \\
\mathrm{b} \\
\mathrm{c} \\
\beta\end{array}$ & $\begin{array}{c}24.5(0.4) \\
6.05(15) \\
22.4(3) \\
111.0(3)\end{array}$ & $\begin{array}{r}-10.6(4.5) \\
2.9(1.6) \\
-14.0(3.5) \\
119.5(4)\end{array}$ & $\begin{array}{c}-4.0(1.1) \\
0.8(0.4) \\
5.5(9) \\
32.7(1)\end{array}$ & $\begin{array}{c}9 \\
13 \\
8 \\
1.5\end{array}$ \\
\hline II & $202-236$ & $\begin{array}{l}\mathrm{a} \\
\mathrm{c}\end{array}$ & $\begin{array}{l}9.4500(45) \\
8.24(16)\end{array}$ & $\begin{array}{c}2.99(2) \\
11.3(1.4)\end{array}$ & $-2.5(3)$ & $\begin{array}{l}6 \\
8\end{array}$ \\
\hline 1 & $236-255$ & $\begin{array}{l}\mathrm{a} \\
\mathrm{c}\end{array}$ & $\begin{array}{l}5.606(9) \\
9.049(15)\end{array}$ & $\begin{array}{l}1.140(35) \\
1.77(6)\end{array}$ & & $\begin{array}{l}8 \\
9\end{array}$ \\
\hline
\end{tabular}

a $\mathrm{R}$ is the reliability factor, $\mathrm{R}^{2}=\left[\left(\sum\left(\mathrm{y}_{0}-\mathrm{y}_{\mathrm{c}}\right)^{2}\right] / \Sigma \mathrm{yc}_{\mathrm{c}}{ }^{2}\right)$, where $\mathrm{y}_{\mathrm{o}}$ and $\mathrm{y}_{\mathrm{c}}$ are the measured and calculated lattice parameters, respectively.

\section{(A)}
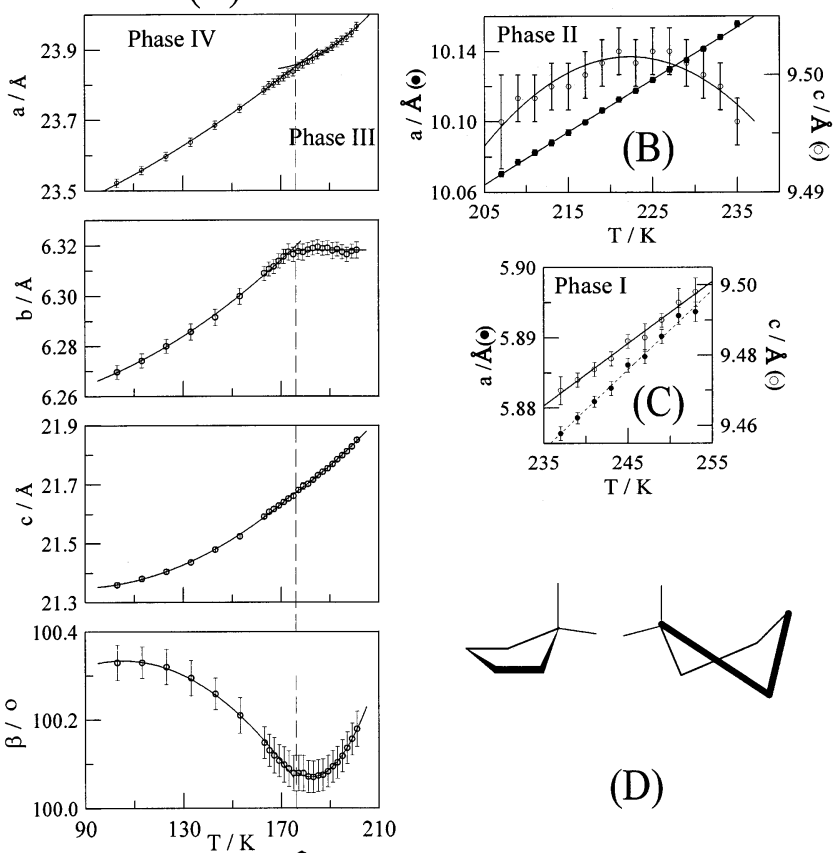

(D)

Figure 1. Lattice parameters of $\mathrm{c}-\mathrm{C}_{5} \mathrm{H}_{10} \mathrm{O}$ for phases IV (T< $176 \mathrm{~K})$ and III (176 K $<\mathrm{T}<202.6 \mathrm{~K})(\mathrm{A})$ and for phases II (B) and I (C) as a function of temperature, and the envelope (left) and the half-chair (right) conformations of $\mathrm{C}_{-} \mathrm{C}_{5} \mathrm{H}_{10} \mathrm{O}$ (D).

density measurements, ${ }^{22} \mathrm{NMR}^{23}$ and X-ray powder diffraction. ${ }^{24}$

The thermodynamic properties concerning the polymorphic behavior of $\mathrm{c}_{-} \mathrm{C}_{5} \mathrm{H}_{10} \mathrm{O}$ corresponding to the stable phases was stated by means of adiabatic calorimetry from 5.4 to $303.0 \mathrm{~K}^{15}$ and can be described as follows. The low-temperature phase IV transforms at $176 \mathrm{~K}$ to phase III with a very low enthalpy change. At

(15) Kabo, G.J .; Diky, V. V.; Kozyro, A. A.; Krasulin, A. P.; Sevruk, V. M. J . Chem. Thermodyn. 1995, 27, 953.

(16) Würflinger, A.; Kreutzenbeck, J .J . Phys. Chem. Solids 1978 39, 193.

(17) Würflinger, A. Ber. Bunsen-Ges. Phys. Chem. 1982, 86, 172

(18) Golfield, G.; Davies, M. Trans. Faraday Soc. 1964, 60, 10.

(19) Green, J . R.; Dalich, S.J .; Griffith, W. T. Mol. Cryst. Liq. Cryst.

1972, 17, 251.

(20) White, A. H.; Bishop, W. S. J . Am. Chem. Soc. 1940, 62, 8.

(21) Davies, M. J. Chim. Phys. 1966, 63, 67.

(22) Wisotki, K. D.; Würflinger, A. J. Phys. Chem. Solids. 1982, $43,13$.

(23) Lambert, J . B.; J ohnson, S. C.; Xue, L.J . Am. Chem. Soc. 1994, 116, 6167.

(24) Green, J . R.; Wheeler, D. R. Mol. Cryst. Liq. Cryst. 1969, 6, 1.
$202.6 \mathrm{~K}$ phase III transforms to the OD phase II. On further heating, OD phase I appears at $234.0 \mathrm{~K}$. Finally the melting temperature is found at $255.6 \mathrm{~K}$ (enthalpy changes are collected in Table 1).

The crystal structures of the OD phases of $\mathrm{c}-\mathrm{C}_{5} \mathrm{H}_{10} \mathrm{O}$ were attempted by Green and Wheeler. ${ }^{24}$ According to the close similarity of the pattern of phase I with that of the OD phase of cyclopentane (hexagonal), they proposed a close-packed hexagonal structure for phase $\mathrm{I}$, in which molecules rotate with spherical symmetry about either its center of mass or either the center of the five-membered ring. F or phase II a hexagonal lattice in which the molecules retain the same positions as in the lattice of phase I, but probably with restricted rotation about some fixed axis, was proposed. It is noteworthy that there is no information available about the lattice structure of the low-temperature ordered phases III and IV. Orientational disorder and appearance of multiple metastable phases together with the existence of non-reconstructive transitions that alter the crystals contributed to such a lack.

Because of the fact that $\mathrm{c}-\mathrm{C}_{5} \mathrm{H}_{10} \mathrm{O}$ has a permanent electric dipole moment and the existence of some orientational freedom in phases II and I, some interesting conclusions were derived from dielectric studies.5,17-21 It was concluded that the high dielectric constant in phase I can be associated with the existence of hydrogenbonded chains which are compatible with a nearly complete orientational freedom of the molecules, giving rise to an almost isotropic solid from a dielectric point of view despite the hexagonal symmetry. ${ }^{19}$ In contrast, the chains in phase II are formed predominantly in the c-direction of the hexagonal lattice, which results in a highly anisotropic solid with regard to the dielectric properties.

An important feature of $\mathrm{c}-\mathrm{C}_{5} \mathrm{H}_{10} \mathrm{O}$ is the possibility of obtaining a glassy crystal when the ODIC state is supercooled to a sufficient extent bel ow the temperature transition to the ordered phase. ${ }^{5,25}$ The glassy state is looked upon as a nonequilibrium state in a thermodynamic sense and as a disordered state when the characteristic dynamical disorder of the OD phase becomes frozen. For cyclic compounds it has been shown that disorder in the glassy crystal phase arises from some degree of disorder in the whole molecular

(25) Dworkin, A.; Fuchs, A. H.; Ghelfenstein, M.; Szwarc, H.J . Phys. Lett. 1982, 43, L-21. 
orientation and from a mixture of conformations due to the difficulties of the system in finding the regular packing in a nonequilibrium state. ${ }^{25,26}$

It is commonly found that the glassy state is obtained after phase I is supercooled. Nevertheless, it should be pointed out that, for many physical properties, such as density, permittivity, or specific heat, the differences between phases I and II are rather small, thus making it difficult to ascertain which of the OD phases of $\mathrm{c}-\mathrm{C}_{5} \mathrm{H}_{10} \mathrm{O}$ was really frozen. In this work we will undoubtedly show that by a fast cooling from the liquid phase II is al ways supercooled, which in fact means that the transition I-II was never bypassed. Such a result has al ready been inferred from di electric experiments. ${ }^{5}$

The purpose of this article is mainly to report on the stable polymorphism of $\mathrm{c}-\mathrm{C}_{5} \mathrm{H}_{10} \mathrm{O}$ and more particularly to characterize from a crystallographic point of view phases IV and III. In addition, the relationships among intermolecular interactions, lattice symmetry, and orientational disorder will be established by means of the study of the thermal-expansion tensor.

\section{Experimental Section}

$\mathrm{C}-\mathrm{C}_{5} \mathrm{H}_{10} \mathrm{O}$, obtained from Aldrich Chemical Co., more than $99 \%$ pure, was carefully purified by means of vacuum vaporization at room temperature. The process was repeated and its efficiency controlled by differential scanning calorimetry (DSC-2920 from TA-I nstruments) until the melting temperature reached a stationary value which, in addition, agreed with the highest reported literature values $\left(255.6 \mathrm{~K},{ }^{15} 256.2 \mathrm{~K}^{16}\right)$.

A set of high-resolution X-ray powder profiles was recorded using a horizontally mounted INEL cylindrical positionsensitive detector (CPS120) ${ }^{27}$ equipped with a liquid-nitrogen INEL CRY 950 cryostat $(80-460 \mathrm{~K})$ with helium gas as the heat exchanger in the sample holder. Temperature accuracy was $1 \mathrm{~K}$ and measurements were performed when fluctuations were lower than $0.3 \mathrm{~K}$. The detector, used in Debye-Scherrer geometry (transmission mode), consisted of 4096 channels and enables a simultaneous recording of the profile over a $2 \theta$ range between 4 and $120^{\circ}$ (angular step of $0.029^{\circ}$ in $2 \theta$ ). Monochromatic $\mathrm{Cu} \mathrm{K} \alpha_{1}(\lambda=1.54059 \AA)$ was selected by means of an asymmetric focusing incident-beam curved quartz monochromator. The generator power was set to $1.2 \mathrm{~kW}$ (40 kV and 30 $m A)$.

The samples were introduced into $0.5-\mathrm{mm}$-diameter Lindemann glass capillaries in the liquid state at room temperature and were rotated perpendicularly to the X-ray beam during data collection to improve averaging of the crystallites.

Patterns were obtained on heating every $10 \mathrm{~K}$ in the temperature range of $93-160 \mathrm{~K}$ and every $2 \mathrm{~K}$ from this temperature up to the melting point, with acquisition times of at least $60 \mathrm{~min}$ and a stabilization time of at least $10 \mathrm{~min}$ at each temperature before the data acquisition.

External calibration by means of cubic phase $\mathrm{Na}_{2} \mathrm{Ca}_{3} \mathrm{Al}_{2} \mathrm{~F}_{14}{ }^{28}$ was performed for channels to be converted into $2 \theta$ degrees by means of cubic spline fittings. The peak positions were determined after pseudo-Voigt fitting by using the PEAKOC application from DIFFRACTINEL software. ${ }^{29}$

(26) Adachi, K.; Suga, H.; Seki, S. Bull. Chem. Soc. J pn. 1972, 45, 1960.

(27) Ballon, J .; Comparat, V.; Pouxe, J . Nucl. Instrum. Methods 1983, 217, 213

(28) Evain, M.; Deniard, P.; J ouanneaux, A.; Brec, R. J . Appl. Crystallogr. 1993, 26, 563.

(29) Masson, O.; Ramponi, A. PEAKOC Program; Laboratoire de Matériaux Céramiques et Traitements de Surfaces, CNRS-ENSCI, Limoges, France, 1996.

\section{Results}

3.1. Phases IV and III. X-ray diffraction profiles of $\mathrm{C}-\mathrm{C}_{5} \mathrm{H}_{10} \mathrm{O}$ were recorded during heating of a sample that was slowly cooled to $93 \mathrm{~K}$ to avoid the appearance of undesirable metastable phases as well as the glassy state. The program DICVOL $91^{30}$ was used to determine the crystal symmetry and lattice parameters of the lowtemperature phase IV. With use of the first 18 strongest reflections of the pattern at $93 \mathrm{~K}$, a monodinic unit cell with $a=23.530(10) \AA, b=6.265(3) \AA, c=21.355(7) \AA$, and $\beta=100.33(3)^{\circ}$ with figures of merit $\mathrm{M}(18)=21.3$ and $F(18)=40.3$ came out. Subsequent lattice parameter refinements using the pattern-matching option of the FULLPROF ${ }^{31}$ program were performed. The systematic absences were consistent with space groups Cc or $\mathrm{C} 2 / \mathrm{c}$

After such an indexing procedure, lattice parameters were refined at each temperature by means of the FULLPROF program. At $123 \mathrm{~K}$ lattice parameters were determined to be $a=23.598(6) \AA, b=6.2801(7) \AA, c=$ $21.405(2) \AA$, and $\beta=100.32(1)^{\circ}$. The set of refined lattice parameters were fitted as a function of temperature (Figure 1 ) by means of a standard least-squares method. Polynomial equations accounting for their variations are gathered in Table 1.

Similarly, the program DICVOL91 was used to de termine crystal symmetry and lattice parameters for phase III at $199 \mathrm{~K}$. With use of the first 19 strongest reflections, a solution close to that found for phase IV (with the same systematic absences) came out with figures of merit: $M(19)=15.0, F(19)=27.5$. Subsequent least-squares refinements by means of the FULLPROF program yiel ded $a=23.949$ (3) $\AA, b=6.3178(7) \AA, c=$ 21.829(2) $\AA$, and $\beta=100.16(1)^{\circ}$ at $199 \mathrm{~K}$.

According to the infrared study, ${ }^{9}$ the clear disappearance of certain bands at the IV to III transition $(24,56$, and $63 \mathrm{~cm}^{-1}$ ) indicates an increase of the symmetry of the asymmetric unit from $C_{2}$ (or $C_{S}$ ) to $C_{2 v}$ together with a small decrease in the strength of the hydrogen bonds. These results are quite compatible with the lattice symmetry of $\mathrm{CC}$ and $\mathrm{C} 2 / \mathrm{C}$ for phases IV and III, respectively. Two experimental diffraction patterns of phases IV and III are depicted in Figure 2 together with patterns simulated by means of the "pattern-matching" option of the FULLPROF program according to the proposed space groups and lattice parameters.

It must be noticed that the sole evidence found for the IV to III transition from X-ray powder measurements has been the small change of the lattice parameters $\mathrm{b}$ and $\beta$ and, still less, for parameters $\mathrm{a}$ and $\mathrm{c}$ as a function of the temperature (see Figure 1).

3.2. OD Phases II and I. Green and Wheeler ${ }^{24}$ determined the lattice symmetry of phase I as hexagonal due to the close similarity between the experimental $\mathrm{X}$-ray diffraction patterns of $\mathrm{c}-\mathrm{C}_{5} \mathrm{H}_{10} \mathrm{O}$ and cyclopentane in their OD phases. In addition, the systematic absences and the c/a ratio (close to 1.633) indicated a close-packed structure with the lattice parameters of $a=5.887 \AA$,

(30) Louër, D.; Boultif, A. DICVOL91 Program; Laboratoire de Cristallochimie, Université de Rennes I: Rennes, France, 1991.

(31) Rodriguez-Carjaval, J . FULLPROF, a program for Rietveld refinement and pattern matching analyses: Abstracts of the satellite meeting on powder diffraction of the $\mathrm{XV}^{\text {th }}$ congress of the International 

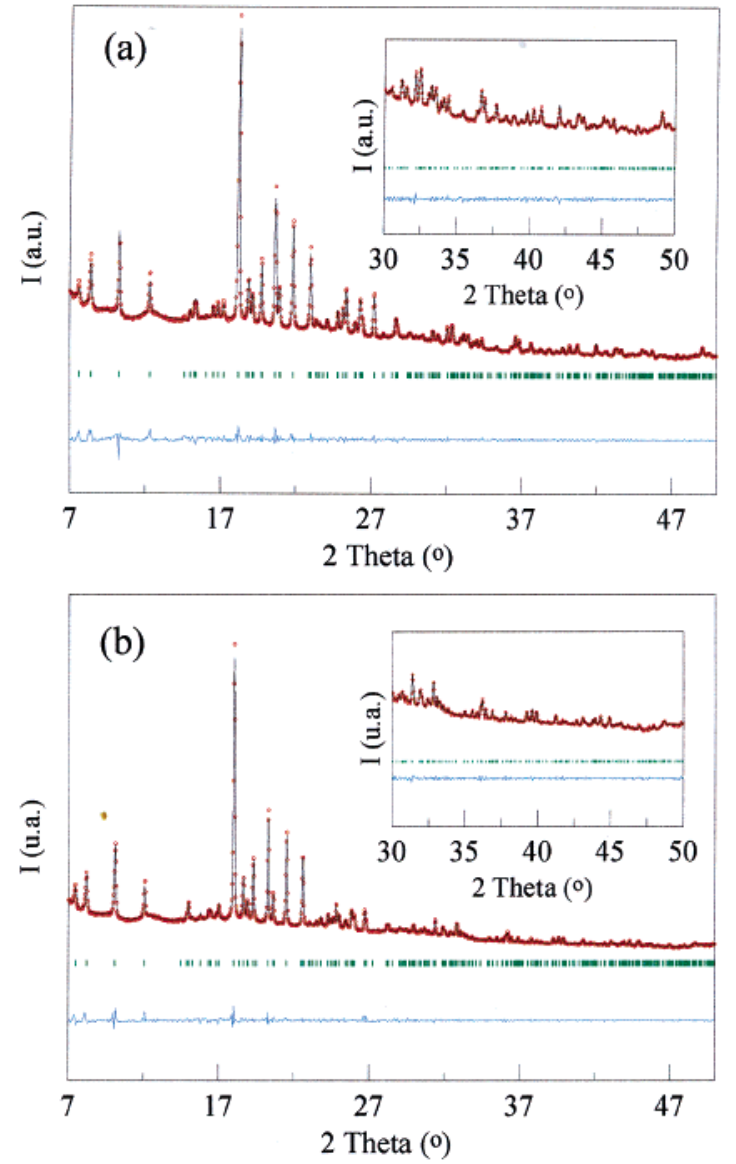

Figure 2. Experimental X-ray diffraction patterns $(O)$ and simulated patterns using the FULLPROF program $(-)$ of phases IV (a) and III (b) for $\mathrm{C}^{-} \mathrm{C}_{5} \mathrm{H}_{10} \mathrm{O}$ at 93 and $199 \mathrm{~K}$ along with the difference profile. (Inset corresponds to a five-times expanded vertical scale for the data from $2 \theta \geq 30^{\circ}$ ).

$c=9.445 \AA$ ( $c / a=1.607)$, and $Z=2$ at $243.2 \mathrm{~K}$. The authors ${ }^{24}$ mentioned above proposed a model in which molecules rotate with "spherical" symmetry around their center of mass. The lattice parameters determined in this work match up those reported previously $(\mathrm{a}=$ 5.883(1) $\AA, c=9.479(2) \AA, c / a=1.611$ at $243.2 \mathrm{~K}$ ).

As far as phase II is concerned, the same authors ${ }^{24}$ reported a hexagonal lattice with the same systematic absences and lattice parameters of $a=10.1 \AA, c=9.49$ $\AA(c / a=0.94)$ and $Z=6$ at $217.2 \mathrm{~K}$. One should bear in mind that despite the similarity between both OD phases II and I, the required point symmetry for lattice II should be lower than that for lattice I, to produce a necessary difference between both phases. From a dynamic point of view this fact means that molecules in phase II do not rotate with a spherical symmetry and that the orientational disorder should correspond to some kind of molecular motion like hindered molecular tumbling or a libration around one or more axis. The accurate lattice parameters found in the present work confirm the values reported previously $(a=10.0996-$ (14) $\AA, c=9.500(2) \AA, c / a=0.94$ at $217.2 \mathrm{~K}$ ).

The lattice parameters as a function of temperature for phases I and II are depicted in Figure 1 and polynomial equations corresponding to the least-squares fits for each parameter are gathered in Table 1.

3.3. Volume Changes. The pressure-temperature phase diagram ${ }^{16}$ enables the calculation of the volume

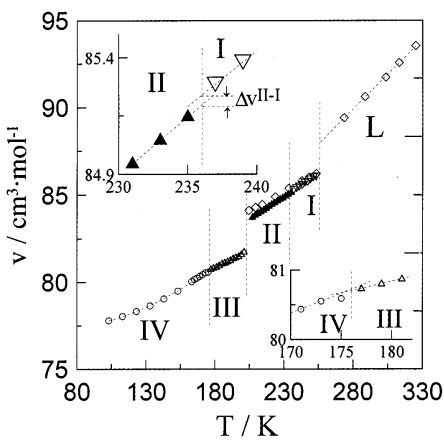

Figure 3. Molar volume of $\mathrm{C}_{-} \mathrm{C}_{5} \mathrm{H}_{10} \mathrm{O}$ as a function of temperature derived from $\mathrm{X}$-ray diffraction experiments for the solid phases and from literature $(\diamond) .{ }^{22}$ Insets correspond to a magnification around temperatures of the IV-III and II-I transitions. $\mathrm{L}=$ liquid.

change at the III to II transition, as well as the $Z$ value of phase III using the molar volume of phase II, the result being $Z=24$. Such a value is also assigned to phase IV, owing to the continuity of lattice parameters.

According to the lattice parameters determined, the molar volume of the solid phases has been plotted in Figure 3. This figure contains also values from the literature corresponding to molar volumes at normal pressure from density measurements as a function of pressure and temperature 22 for the OD phases I and II as well as for the liquid state. As can be seen, the agreement between both sets of values is rather good, the maximum difference in phase II (0.6\%) appearing at temperatures close to the III-II transition and being certainly within the experimental error limits. For phase I the maximum difference is $0.3 \%$.

The volume changes at the transitions have been calculated by extrapolating the molar volumes at the transition temperatures. The results are compiled in Table 2.

Volume changes accompanying the phase transitions (insets in Figure 3 show the details for the volume change at the IV $-\mathrm{III}$ and II-I transitions) have also been calculated using the Clausius-Clapeyron equation. The slopes of the transition lines $d T_{d} d p$ at normal pressure were taken from ref 16 , while the enthalpy changes were obtained from the most recent work of Kabo et al. ${ }^{15}$

Despite the similarity between the molar volumes of phases II and I, a small but substantial volume change appears at the transition temperature. In contrast, volumes for phases IV and III at $176 \mathrm{~K}$ look virtually identical, giving rise to a zero volume change at this transition.

3.4. Glassy State. The nonergodic (or glassy or "orientational glass", OG) state corresponding to an OD phase of a compound should be experimentally available by means of a rapid and low enough temperature quench. 3,32,33 Such a state appears when the reorienting molecules of the OD phase freeze into random configurations after quenching. From a structural point of view, it is evident that thermal contraction diminishes the number of sterically compatible local structures, giving rise to some changes in the orientational molecular correlations. From a dynamical point of view, the glass

(32) Suga, H.; Seki, S. J . Non-Cryst. Solids 1974, 16, 171.

(33) Höchli, U. T.; Knorr, K.; Loidl, A. Adv. Phys. 1990, 39, 405. 
Table 2. Temperature Transitions $T^{*}$ and Enthalpy Changes $\Delta H^{*}$ Derived from the Adiabatic Calorimetry Measurements at Normal Pressure from Kabo et al.; ${ }^{15}$ the Slope of the Temperature-Pressure Two-Phase Equilibria dT/dp Derived from Thermal Analysis at High-Pressure from Wurflinger et al. ${ }^{16}$ and Volume Changes Determined from Density Data as a Function of Pressure and Temperature $\left(\Delta \mathrm{v}^{\mathrm{PvT}}\right){ }^{22}$ from the Clausius-Clapeyron Equation ( $\left.\Delta \mathrm{v}^{\mathrm{CC}}\right)$ and from X-ray Powder Diffraction Measurements $\left(\Delta v^{X R}\right)$ for the Different Transitions of $c-C_{5} H_{10} O^{a}$

\begin{tabular}{|c|c|c|c|c|c|c|c|}
\hline & $\begin{array}{l}\mathrm{T} \\
\mathrm{K}\end{array}$ & $\begin{array}{l}\mathrm{T}^{*} \\
\mathrm{~K}\end{array}$ & $\begin{array}{c}\Delta \mathrm{H}^{*} \\
\mathrm{~J} \cdot \mathrm{mol}^{-1}\end{array}$ & $\begin{array}{c}(\mathrm{dT} / \mathrm{dp}) \\
\mathrm{K} \cdot \mathrm{MPa}^{1-}\end{array}$ & $\begin{array}{c}\Delta \mathrm{v}^{\mathrm{PvT}} \\
\mathrm{cm}^{3} \cdot \mathrm{mol}^{-1}\end{array}$ & $\begin{array}{c}\Delta \mathrm{v}^{\mathrm{CC}} \\
\mathrm{cm}^{3} \cdot \mathrm{mol}^{-1}\end{array}$ & $\begin{array}{c}\Delta \mathrm{v}^{\mathrm{XR}} \\
\mathrm{cm}^{3} \cdot \mathrm{mol}^{-1}\end{array}$ \\
\hline $\mid \mathrm{IV}-\mathrm{III}$ & $176 \pm 1$ & 176 & $57 \pm 5$ & & & & $\approx 0$ \\
\hline$|I|-||$ & $202 \pm 1$ & 202.6 & $3366 \pm 14$ & 0.12 & $3.13^{\#}$ & 1.99 & $1.75 \pm 0.08$ \\
\hline $11-1$ & $236 \pm 1$ & 234.0 & $55 \pm 6$ & 0.10 & 0.06 & 0.024 & $0.032 \pm 0.06$ \\
\hline$I-L$ & $256 \pm 1$ & 255.6 & $1227 \pm 5$ & 0.34 & 2.4 & 1.63 & $1.78 \pm 0.12$ \\
\hline
\end{tabular}

a Temperature transitions from this work are also displayed $(T)$. Volume changes from density data as a function of $p$ and $T$ were obtained in ref 22 by extrapolating the volume changes as a function of pressure to $1 \mathrm{~atm}$. (The value denoted by ${ }^{\#}$ was assigned to the metastable III-II transition at $195 \mathrm{~K}$ ).

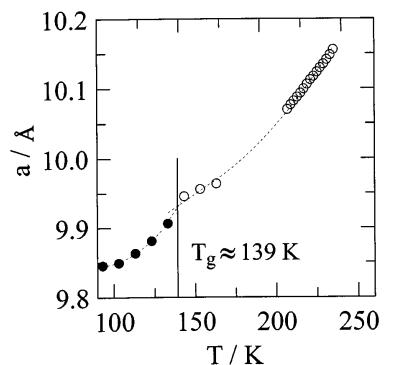

Figure 4. Lattice parameter a of the hexagonal phase II as a function of temperature after quenching from the liquid state.

transition appears when well-defined large amplitude motions are frozen. When heating the OG state, the transition to its ergodic state exhibits all the characteristics observed in a structural-glass transition.

In the case of $\mathrm{c}-\mathrm{C}_{5} \mathrm{H}_{10} \mathrm{O}$, such a quench was performed, as usual, from the liquid state down to about $93 \mathrm{~K}$. The patterns obtained at the lowest temperature for the quenched samples were virtually identical to those corresponding to the stable OD phase II and, thus, undoubtedly indexed as such. In addition, because no additional reflection of the hexagonal lattice of phase II becomes observable in the OG state, it can be concluded that the local disorder of phase II is kept in the OG state. These results mean that the transitions from liquid to phase I and from I to II were not bypassed despite the immersion of the Lindemann capillaries into liquid nitrogen.

Figure 4 depicts the lattice parameter a as a function of temperature after the melt was quenched. The continuous variation of the lattice parameter vs temperature and the discontinuity on the vol ume expansion that would appear due to the change of the slope of a vs $T$ at $T_{g}(\approx 139 \mathrm{~K})$ are irrefutable signatures of the existence of the OG state. ${ }^{34}$ On continuously increasing temperature after the glass transition occurred, the supercooled phase II still remains up to $163 \mathrm{~K}$, temperature at which the sample falls down to phase IV.

3.5. Thermal-Expansion Tensor. The cell deformation $\mathrm{dU}$ due to a small temperature variation $\mathrm{dT}$ is expressed by a second-rank tensor $\mathrm{dU}_{\mathrm{ij}}=\alpha_{\mathrm{ij}} \mathrm{dT}$, where $\alpha_{i j}\left(K^{-1}\right)$ are the coefficients of the thermalexpansion tensor. At a given temperature, knowledge of the principal coefficients and of the direction of the principal axes of the tensor allows the determination

(34) Tamarit, J . LI.; López, D. O.; de la Fuente, M. R.: PérezJ ubindo, M. A.; Salud, J .; Barrio, M. J. Phys. Condens. Matter 2000, 12,8209 .

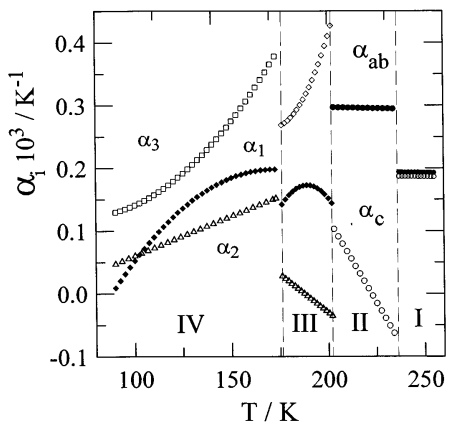

Figure 5. The $\alpha_{i}$ principal coefficients as a function of temperature for $\mathrm{C}-\mathrm{C}_{5} \mathrm{H}_{10} \mathrm{O}$. For phases IV and III $\alpha_{2}$ is parallel to the crystallographic $b$ direction in the monoclinic phase. For the hexagonal phases II and I, $\alpha_{a b}$ and $\alpha_{c}$ corresponds to the principal coefficients in the (001) plane and along the [001] crystallographic direction.

of the strongest and the weakest directions of the corresponding intermolecular interactions. ${ }^{10,35}$ Such directions are referred to as "hard" and "soft" directions, respectively. ${ }^{10,36-38}$ The procedure and the method to determine the tensor from the lattice parameters variation with temperature have been published elsewhere $\mathrm{e}^{10}$ and the program DEF ORM ${ }^{39}$ was used for the calculation of the tensor.

For a monoclinic lattice, the tensor is completely defined by the principal coefficients, $\alpha_{1}, \alpha_{2}$, and $\alpha_{3}$, and an angle $\varphi$ between the direction of one of the principal directions ( $\alpha_{3}$ in the present case) and the crystallographic axis a, the $\alpha_{2}$ axis being coincident with the 2-fold axis b of the crystal.

According to the Newmann principle, for which the thermal-expansion tensor has to display the point group symmetries of the crystal, the 3-fold hexagonal axis c must be a revolution axis of the tensor; that is, the intermolecular interactions in the (00I) plane are independent of the direction. Consequently, the principal coefficients for a hexagonal lattice can be reduced to two nondependent directions: $\alpha_{c}$, which is parallel to the crystall ographic c direction and $\alpha_{a b}$, which accounts for the thermal expansion in any direction of the ( $\mathrm{OOI})$ plane.

(35) Garnier, P.; Calvarin, G.; Weizel, D. J . Chim. Phys. Phys.-Chim Biol. 1972, 11-12, 1711.

(36) Tamarit, J . LI.; Barrio, M.; López, D. O.; Haget, Y. J . Appl. Crystallogr. 1997, 30, 118.

(37) Tamarit, J . LI.; López, D. O.; Alcobé, X.; Barrio, M.; Salud, J .; Pardo, L. C. Chem. Mater. 2000, 12, 555-563.

(38) Negrier, P.; Pardo, L. C. Salud, J .; Tamarit, J . LI.; Barrio, M.; López, D. O.; Würflinger, A.; Mondieig, D. Chem. Mater. 2002, 14, 1921.

(39) Filhol, A.; Lajzerowicz, J.; Thomas, M. DEFORM program, unpublished software, 1987. 

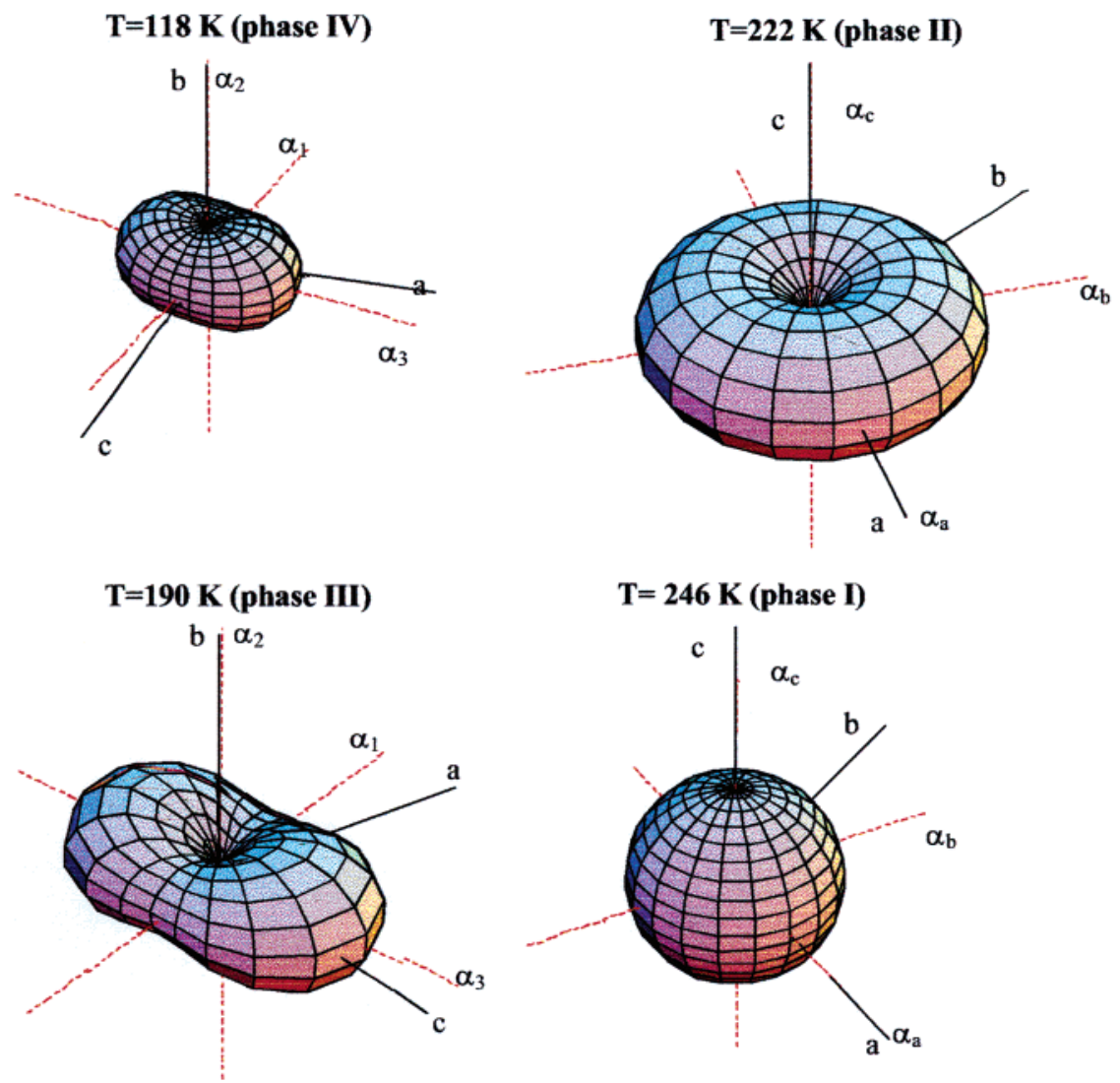

Figure 6. Three-dimensional thermal-expansion tensors of monoclinic phases IV and III at 118 and $190 \mathrm{~K}$, respectively, and of the hexagonal phases II and I at 222 and $246 \mathrm{~K}$, respectively, for $\mathrm{c}_{-} \mathrm{C}_{5} \mathrm{H}_{10} \mathrm{O}$ al ong its principal directions $\alpha_{1}, \alpha_{2}$, and $\alpha_{3}$ (dotted red line) together with the crystallographic axes $a, b$, and c (continuous back line). The full length of the $\alpha_{i}$ axes corresponds to $10^{-4} \mathrm{~K}^{-1}$.
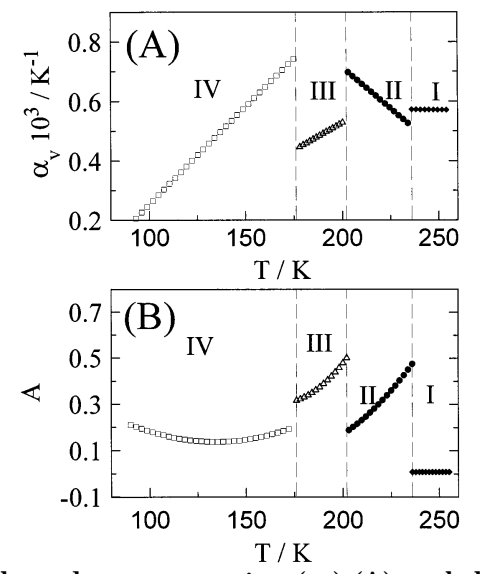

Figure 7. The volume expansion $\left(\alpha_{v}\right)(A)$ and the aspherism index (B) as a function of the temperature for phases IV, III, $\mathrm{II}$, and I of $\mathrm{C}-\mathrm{C}_{5} \mathrm{H}_{10} \mathrm{O}$.

The variations of the principal coefficients $\alpha_{i}$ with temperature for $\mathrm{c}-\mathrm{C}_{5} \mathrm{H}_{10} \mathrm{O}$ are plotted in Figure 5. The three-dimensional thermal-expansion deformation tensor at different temperatures for phases IV, III, II, and I along its principal axes together with the direction of the crystallographic axes are sketched in Figure 6.

The vol ume expansion $\alpha_{V}$, cal culated as $\alpha_{V}=\alpha_{1}+\alpha_{2}$ $+\alpha_{3}$, is shown in Figure 7A as a function of temperature for all solid phases.

It can be clearly seen that volume expansion for phase IV increases with temperature and a strong decrease is found at the transition IV to III. Such a discontinuity is mainly due to the change of the $\alpha_{2}$ principal coefficient (which corresponds to the 2-fold crystallographic b axis) with the temperature and provides an additional proof for the IV to III transition.

Concerning the orientationally disordered phase II, volume expansion decreases (the phase becomes harder) when temperature increases. This particular behavior is produced by the contraction of the hexagonal lattice II in the crystallographic c direction.

At this point it should be emphasized that any analysis of the volume expansion neglects the details corresponding to the specific intermolecular interactions and thus all the details about a possible anisotropy. To account for this anisotropy, the aspherism index $\mathrm{A}=$ $(2 / 3)\left[1-\left(3 \beta / \alpha v^{2}\right]\right)^{1 / 2}$, where $\beta=\alpha_{1} \alpha_{2}+\alpha_{2} \alpha_{3}+\alpha_{1} \alpha_{3}$, has been defined. ${ }^{40} \mathrm{~F}$ igure $7 \mathrm{~B}$ shows this index as a function of temperature. It can be clearly observed that such a parameter is almost zero for phase I despite the hexagonal symmetry. In contrast, for hexagonal phase II the aspherism increases with temperature, making evident the increase of the asymmetry of the intermolecular interactions.

\section{Discussion}

The IV to III transition was earlier reported by Parks et al. ${ }^{14}$ to be at $162 \mathrm{~K}$, which clearly disagrees with the value found in the most recent work of Kabo et al. (176 K). ${ }^{15}$ Such a difference is probably related to a wrong interpretation of a specific heat anomaly in the original measurements of Parks et al. The lattice parameter

(40) Weigel, D.; Beguemsi, T.; Garnier, P.; Gerad, J . F. J . Solid State Chem. 1978, 23, 24. 
variation in the temperature range covering phases IV and III (Figure 1) shows a slight change at a temperature close to $176 \mathrm{~K}$, thus confirming the most recent work of adiabatic calorimetry. ${ }^{15}$ As for the space groups assigned to phases IV and III (CC and C2/C), it is clear that, in addition to the compatibility between them and the results inferred from infrared spectroscopy mentioned previously, simple symmetry reasons are claimed for such an assignment. This means that at the IV to III transition only slight changes in the orientation of the molecule have occurred, in such a way that if the lattice symmetry of both phases is related by a groupsubgroup relationship, it would be quite possible that the difference of the respective heat capacities would be very low, as has been reported. A similar case has been recently described for the low-temperature phases (III and II) of $\left(\mathrm{CH}_{3}\right)_{2} \mathrm{CCl}_{2}$, for which the transition between phases III and IV is an ordering transition between $\mathrm{C} 2 / \mathrm{C}$ and $\mathrm{Cc}$ symmetry groups. ${ }^{38}$ Finally, it must be noted that this line of reasoning is compatible with the low entropy change measured for the IV - III transition $\left(0.324 \mathrm{~J} \cdot \mathrm{K}^{-1} \cdot \mathrm{mol}^{-1}\right){ }^{15}$ as well as with the virtually equal volumes for both phases at the transition (Figure 3).

The changes in the lattice parameters with temperature provide noticeable changes in the thermal-expansion tensor. The principal direction $\alpha_{2}$, which is the hard direction (the strongest intermolecular interactions) for phase IV at temperatures close to the IV to III transition, becomes still harder for phase III (see Figure 5). In addition, the soft directions (the weakest intermolecular directions) $\alpha_{1}$ and $\alpha_{3}$, which bel ong to the (0k0) crystallographic plane for both phases, change slightly their respective orientations in such a way that the largest expansion is close to the crystallographic a direction for phase IV and to the crystallographic c direction for phase III. This increase of the intermolecular interactions in the principal direction $\alpha_{2}$ for phase III is accompanied also by a hardening of the $\alpha_{3}$ direction, giving rise to a decrease of the volume expansion (Figure 7A). Although more details of the structure of phases IV and III are required to go further into the particularities of the intermolecular interactions, the aspherism index (Figure 7B) evidences a decrease of the symmetry of spatial distribution of such interactions on going from phase IV to phase III.

In an early work concerning the dielectric properties of $\mathrm{c}-\mathrm{C}_{5} \mathrm{H}_{10} \mathrm{O}$, Davies ${ }^{21}$ suggested that four molecules are involved by hydrogen bonds in a cyclic pattern for phase I. Such a number, together with the lattice symmetry of the phase in which molecules are rotating with near spherical symmetry, ${ }^{24}$ would require a high orientational disorder. Thus, the principal coefficients of the tensor in such a phase are quite similar, giving rise to an aspherism index which reaches almost the zero value for phase I and then revealing a high isotropy for the intermolecular interactions.

In contrast, for phase II the existence of linear hydroxyl chains has been pointed out. ${ }^{21}$ According to our results, the noticeable anisotropy of the thermal-expansion tensor of phase II, revealed by the large value of the aspherism index (Figure 7B), points out that hydrogen-bonding interactions are essentially in the $C$ direction, thus being the [001] crystallographic direction corresponds to the hard direction while the (00I) plane corresponds to the soft one. It must be noted that in the $c$ direction of phase II the $\alpha_{c}$ coefficient (Figure 5) shows even negative values, which in fact means that even a contraction takes place at temperatures close to the II to I transition. Such an anisotropy of the intermolecular interactions evidences that the rotation of the molecules in phase II is probably restricted to rotation about some fixed axes or to a libration around the 6-fold axis of the hexagonal lattice. At the transition to phase I such a librational disorder would increase the amplitude in such a way that it would become al most isotropic and the symmetry of the new phase (for which the translational order is virtually the same as that of the parent phase II) increases.

As far as volume changes are concerned, it should be noted that the values calculated from the X-ray diffraction measurements are virtually the same as those calculated from the Clausius-Clapeyron relation for which enthal py values of ref 15 and $\mathrm{dT}_{\mathrm{C}} / \mathrm{dp}$ slopes from ref 16 have been used. Large discrepancies are found when compared to the values obtained from highpressure dilatometric measurements. ${ }^{22}$ In this case it should be mentioned that the normal pressure values (here summarized) are obtained from the extrapolation of high-pressure densities and therefore probably submitted to a high error.

\section{Conclusions}

The polymorphic behavior of $\mathrm{c}_{-} \mathrm{C}_{5} \mathrm{H}_{10} \mathrm{O}$ has been studied from $93 \mathrm{~K}$ to the liquid state by means of $\mathrm{X}$-ray powder diffraction measurements.

Lattice symmetry of the low-temperature phases IV and III have been determined as monoclinic (space group Cc and C2/c, respectively), both with 24 molecules per unit cell. Such a large number, together with the existence of conformational disorder, makes it difficult to determine the structure of both phases.

In addition, the IV to III phase transition, previously found throughout heat capacity measurements, ${ }^{15}$ has been stated. The si milarity between lattice parameters of both phases at the transition temperature gives rise to a continuous variation of the molar volume throughout the IV to III transition.

As for the orientationally disordered phases II and I, hexagonal symmetry has been confirmed for both phases and the lattice parameters have been car efully determined as a function of temperature.

Despite the disorder, the existence of weak preferred orientations of molecules in phase II has been evidenced by means of the high ani sotropy of the thermal-expansion tensor and, more particularly, by the high value of the aspherism index. In contrast, phase I reveals high isotropy with regard to the thermal-expansion tensor and thus, as far as intermolecular interactions are concerned, it behaves almost as a cubic phase.

Acknowledgment. This work has been performed by the Spanish DGE under Grant PB98-0923 and supported by the Grant SGR2002-00152 of the Catalan government. 